\title{
Two-photon Fluorescence Endomicroscopy
}

\author{
Yicong $\mathrm{Wu}$ and Xingde $\mathrm{Li}$ \\ Department of Biomedical Engineering, Johns Hopkins University \\ Baltimore, Maryland 21205 \\ U.S.A.
}

\section{Introduction}

Two-photon fluorescence (TPF) microscopy is a powerful technique for high-resolution imaging of biological tissues, enabling depth-resolved morphological and functional assessment of biological tissues via a non-invasive route (Denk et al., 1990; Helmchen \& Denk, 2005; Konig, 2000; Zipfel et al., 2003). In TPF microscopy, a molecule (i.e., the fluorophore) can absorb two photons quasi-simultaneously $\left(10^{-15}-10^{-18} \mathrm{~s}\right)$ and emit a single photon during relaxation from the excited state to the ground state. The probability for the fluorescent emission is thus quadratically dependent on the excitation light intensity. With a focusing unit, much more two-photon fluorescence is generated from the focal spot than where the beam is diffused. Effectively, excitation is restricted to the very small focal volume ( 1 femtoliter), resulting in the inherent optical sectioning ability without the need for a pinhole to reject out-of-focus photons. This optical sectioning capability permits wholefield fluorescence collection and thus enhances the collection efficiency in highly scattering tissues. In TPF microscopy, near-infrared (NIR) femtosecond laser is generally employed for effective excitation, which increases the penetration depth and reduces image deterioration due to the less scattering in turbid tissues. In addition, NIR excitation likely causes less photodamage outside the focal volume.

With the advances in micro-optics and micro-mechanical components, a TPF endomicroscopy system is becoming attractive as a basic research tool with a much smaller form factor and lower cost compared to a conventional TPF microscope. Moreover, the TPF endomicroscopy system has a great potential to transform the powerful TPF technology for in vivo studies and clinical applications. Recently, increasing interests have been focusing on the development of TPF endomicroscope with a small size which can go through the accessory port of a standard endoscope for in vivo and clinical studies while maintaining the TPF imaging ability similar to a standard TPF microscope. Major challenges for TPF endomicroscopy devices are efficient delivery of single-mode ultrashort pulses, wide-field collection of the TPF signals, fast 2-D/3-D beam scanning with a miniature objective lens of good optical properties, and overall miniaturization of the probe assembly (Bao et al., 2008; Engelbrecht et al., 2008; Flusberg et al., 2005a; Flusberg et al., 2005b; Fu et al., 2006; Gobel et al., 2004a; Helmchen et al., 2001; Hoy et al., 2008; Jung \& Schnitzer, 2003; Jung et al., 2008; Konig et al., 2007; Le Harzic et al., 2008; Levene et al., 2004; Myaing et al., 2006; Wu et al., 2009a; Wu et al., 2009b).

Source: Advances in Lasers and Electro Optics, Book edited by: Nelson Costa and Adolfo Cartaxo, ISBN 978-953-307-088-9, pp. 838, April 2010, INTECH, Croatia, downloaded from SCIYO.COM 
This book chapter offers a review of fiber-optic TPF endomicroscopy technologies with emphasis on major technological development challenges. The advantages and limitations associated with various TPF endomicroscopy systems are discussed. Special design and engineering considerations are presented with our recently developed all-fiber-optic rapid scanning TPF imaging endomicroscopy system as an example. Some representative endomicroscopic TPF imaging results are illustrated, demonstrating that the emerging TPF endomicroscopy systems are very promising for basic laboratory research and for early disease detection and image-guided interventions.

\section{Challenges in two-photon fluorescence endomicroscopy}

\subsection{Single-mode femtosecond laser delivery and large-area TPF signal collection}

The first major issue in TPF endomicroscopic implementation is how to efficiently deliver single-mode femtosecond excitation light and collect multimode two-photon fluorescence signals. It is well known that a single-mode fiber (SMF) can be used to deliver and focus single-mode femtosecond excitation light to a near diffraction limited spot. However, the TPF collection efficiency severely suffers due to the small core diameter of a SMF. Some embodiments utilize a separate multimode fiber for effective TPF collection (Helmchen et al., 2001), as shown in Fig. 1(a). The multimode fiber with large core diameter (e.g. 1-2 mm) and high NA (e.g. 0.4-0.8) increases the collection area and it also makes the collection efficiency less sensitive to the spherical and chromatic aberration of the objective lens. Such configuration can be further improved by replacing the common SMF with a hollow-core photonic bandgap fiber (HC-PCF) with zero dispersion at the selected excitation wavelength (Engelbrecht et al., 2008; Flusberg et al., 2005b; Gobel et al., 2004b; Hoy et al., 2008; Le Harzic et al., 2008). Owing to the dramatically reduced group-velocity dispersion (GVD) and nonlinear optical effects (such as self-phase modulation, SPM) in the specially designed HC$\mathrm{PCF}$, femtosecond pulses in HC-PCF experience negligible temporal distortion, and no additional pulse prechirping is required (Agrawal, 2007).
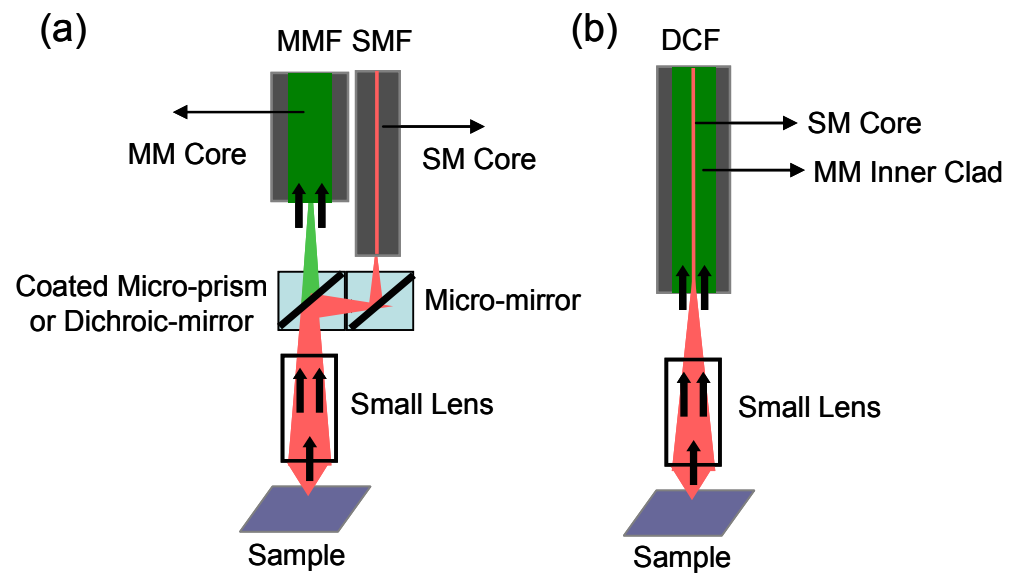

Fig. 1. Schematic of fiber-optic two-photon fluorescence endomicroscope: (a) Two-fiber configuration; (b) Single-fiber configuration. SMF: single-mode fiber; MMF: multi-mode fiber; DCF: double-clad fiber; SM: single-mode; MM: multi-mode. 
As shown in Fig. 1(a), the two-fiber configuration involves a dichroic mirror and a prism and it is difficult to minimize the endomicroscope. In order to create a more compact and flexible endomicroscope, single double-clad fibers (DCFs, see Fig. 2) have been employed in TPF endomicroscopes for both single-mode laser excitation delivery with the single-mode core and efficient TPF collection with the multimode inner cladding layer (Bao et al., 2008; Fu et al., 2007; Fu et al., 2006; Jung et al., 2008; Myaing et al., 2006; Wu et al., 2009a; Wu et al., 2009 b), as shown in Fig. 1(b). With the advance of fiber fabrication technology, double-clad fiber could be developed with high performance including large inner clad diameter and NA and less nonlinear optical effects.

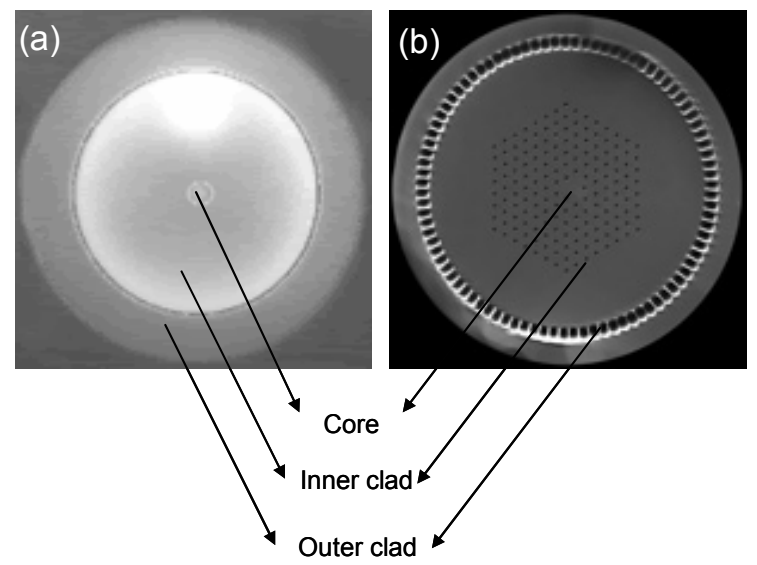

Fig. 2. Schematic of double-clad fibers generally employed in two-photon fluorescence endomicroscopy systems: (a) Conventional double-clad fiber; (b) Photonic crystal doubleclad fiber.

A conventional DCF, as shown in Fig. 2(a), is a step-index fiber composed of a single-mode core, a multi-mode inner cladding layer and an outer cladding layer. The materials for the three layers are typically germanium-doped silica, pure silica and fluorine-doped silica, respectively. The DCF, allowing single-mode delivery of fs excitation light through the single-mode core and collection of multimode TPF signals via the inner clad, is commercially available (Fibercore Ltd., SMM900) and has been successfully implemented in a scanning fiber-optic TPF endomicroscope with an excellent imaging ability (Bao et al., 2008; Myaing et al., 2006; Wu et al., 2009a; Wu et al., 2009b). Compared to a single-mode fiber, the DCF (with core/inner clad diameter of 3.5/103 $\mu \mathrm{m}$ and NA of 0.19/0.24) greatly improves the collection efficiency of TPF signals by 2-3 orders. Another type of DCF is photonic crystal double-clad fiber (PC-DCF) as shown in Fig. 2(b). The PC-DCF comprises a single-mode core with pure silica and inner and outer cladding layers with hybrid air-silica structures (Bjarklev et al., 2003; Knight, 2003). PC-DCF is also commercially available (Crystal Fiber, DC-165-16-Passive) and has been used for developing TPF endomicroscopy technologies (Fu et al., 2005; Fu et al., 2007; Fu et al., 2006; Jung et al., 2008). The PC-DCF has a core/inner clad diameter of $16 / 165 \mu \mathrm{m}$ and NA of 0.04/0.6. The large core of the PC-DCF reduces the nonlinear optical effects up to a certain excitation power (Bao \& $\mathrm{Gu}, 2009$ ). But the large core diameter and the related low NA make it challenging to focus the excitation beam to a small spot size with a given miniature objective lens. The use of a PC-DCF would 
also increase the rigid length of an endomicroscope at its distal end due to the requirement of beam expansion and refocusing mechanisms. Generally speaking, in engineering a compact fiber-optic TPF endomicroscope, the core size of the DCF has to be carefully chosen with a tradeoff among the excitation/collection efficiency, the nonlinear effects, the overall diameter and the rigid length of the probe.

Since the SMF, DCF and PC-DCF have normal dispersion, ultrashort pulses transmitting in these fibers will be temporally broadened due to GVD and nonlinear effects such as SPM (Agrawal, 2007), resulting in the reduction of TPF excitation efficiency. Therefore, prechirping is required for fiber-optic TPF endomicroscopes with such fibers. A conventional pulse stretcher based on a grating and lens pair can be utilized for negative prechirping before the pulses are launched into the fibers (Bao et al., 2008; Helmchen et al., 2001; Myaing et al., 2006; Treacy, 1969). However, the grating/lens pulse stretcher consists of bulky optics with a double-pass configuration which is generally sensitive to alignment and has suboptimal throughput. Recently, photonic crystal fibers based on photonic bandgap effects to guide light propagation have been developed. These fibers exhibit anomalous dispersion over certain wavelength range and can be used for prechirping (Bjarklev et al., 2003; Reeves et al., 2003). For example, the hollow-core photonic bandgap fiber (PBF) from Crystal Fibre (HC-800-02) offers negative GVD with the wavelength longer than $800 \mathrm{~nm}$. It has been employed for dispersion compensation in the endomicroscopes (Wu et al., 2009a; Wu et al., $2009 \mathrm{~b})$. Table 1 summarizes the measured GVD parameter $\left(\beta_{2}\right)$ and dispersion parameter (D) for excitation pulses at $810 \pm 18 \mathrm{~nm}$ with an initial pulse width of $60 \mathrm{fs}$. The reference values of a conventional silica core single-mode fiber (SMF) at $810 \mathrm{~nm}$ are listed (Agrawal, 2007). As can be seen, the measured GVD of the DCF is $\sim 43,065 \mathrm{fs}^{2} / \mathrm{m}$, whereas the PBF offers a negative GVD of $\sim 35,246 \mathrm{fs}^{2} / \mathrm{m}$. As a result, the positive dispersion of a DCF can be compensated by a PBF when the length ratio of the PBF to DCF is $\sim 1.1$ at $810 \pm 18 \mathrm{~nm}$. The achievable pulse width is about $130 \mathrm{fs}$ with $20 \mathrm{~mW}$ delivered through the DCF core. As the power laser transmitting in the DCF core increases (e.g. up to $50 \mathrm{~mW}$ ), the pulses suffer selfphase modulation and other nonlinear effects, and the temporal pulse duration broadens to about $200 \mathrm{fs}$.

\begin{tabular}{ccc}
\hline & $\beta_{2}\left(\mathrm{fs}^{2} / \mathrm{m}\right)$ & $\begin{array}{c}\mathrm{D} \\
(\mathrm{ps} / \mathrm{nm} / \mathrm{km})\end{array}$ \\
\hline DCF $(810 \pm 18 \mathrm{~nm})$ & 43,065 & -123.7 \\
PBF $(810 \pm 18 \mathrm{~nm})$ & $-35,246$ & 101.2 \\
SMF $(810 \mathrm{~nm})^{*}$ & 34,120 & -98.0 \\
\hline
\end{tabular}

Table 1. Measured GVD parameter $\left(\beta_{2}\right)$ and dispersion parameter $(D)$ of a conventional double-clad fiber (DCF) and hollow-core photonic bandgap fiber (PBF). For reference, the $\beta_{2}$ and D values of a single-mode fiber (SMF) are cited from Ref. (Agrawal, 2007).

\subsection{Miniature high-speed scanning head}

The second challenge in developing a fiber-optic TPF endomicroscope is the beam scanner at the distal end which has to be in a small footprint. Current endomicroscope embodiments are mainly based on micro-electro-mechanical system (MEMS) scanning mirrors (Bao et al., 2008; Fu et al., 2006; Hoy et al., 2008; Jung et al., 2008; Piyawattanametha et al., 2006) (Fig. 3(a)) and piezoelectric resonant fiber-optic scanners (Engelbrecht et al., 2008; Flusberg et al., 2005b; Helmchen et al., 2001; Myaing et al., 2006; Wu et al., 2009a; Wu et al., 2009b) (Fig. 
3(b)). 1-D or 2-D scanning mirrors can be micro-fabricated on a single silicon plate with torsional hinges, supporting substrates and control circuits integrated on the same chip (Hagelin \& Solgaard, 1999; Lin \& Fang, 2003; Yao \& MacDonald, 1997). The use of electrostatic actuation, in particular those with a comb drive structure, permits low power consumption and strong actuation force (Hah et al., 2004). A wide range of frequency response from $100 \mathrm{~Hz}$ to $10 \mathrm{kHz}$ can be achieved with MEMS scanners. Typical MEMS mirrors with a $0.5-2 \mathrm{~mm}$ diameter can have a mechanical scanning angle up to $\sim 30^{\circ}$ with reasonably low driving voltages ( 10-120 V) (Lang et al., 1999; Schenk et al., 2000). Using MEMS techniques, a raster scanning pattern can be easily created, as shown in Fig. 3(a). Overall, MEMS scanners have a great potential to be integrated in a compact endomicroscope yet the relatively large substrates with the drive circuits still present significant engineering challenges in their endomicroscopic applications. A TPF endomicroscope based on a 2-D MEMS mirror with a size of $\sim 3.2 \mathrm{~mm} \times 3 \mathrm{~mm}$ has been firstly developed with a line acquisition rate of $3.5 \mathrm{kHz}$ (Piyawattanametha et al., 2006). Later, another 2-D MEMS mirror with a size of $\sim 5 \mathrm{~mm}$ in diameter, with a speed of 7 lines/s over an area of $80 \times 130 \mu \mathrm{m}^{2}$ has been assembled in a TPF endomicroscope (Fu et al., 2007). Recently, higher TPF imaging rate up to 10 frames per second has been demonstrated in an endomicroscope prototype but with a large dimension of $10 \times 15 \times 40 \mathrm{~mm}^{3}$ (Hoy et al., 2008).

(a)
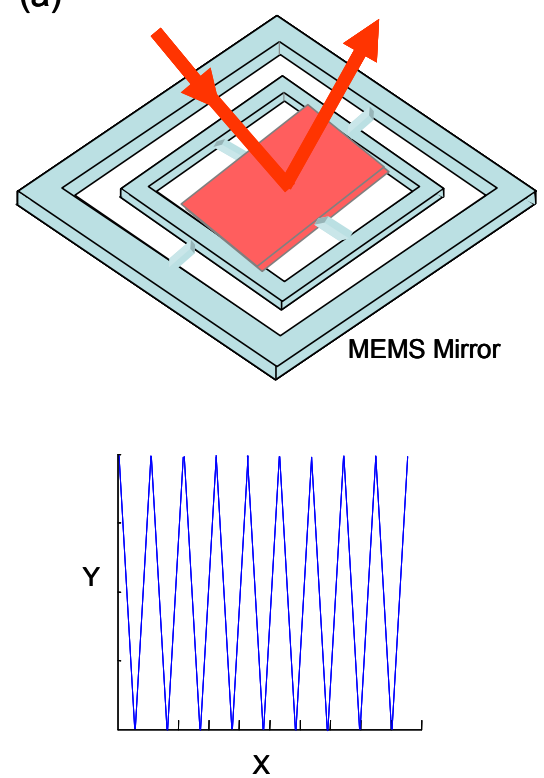

(b)
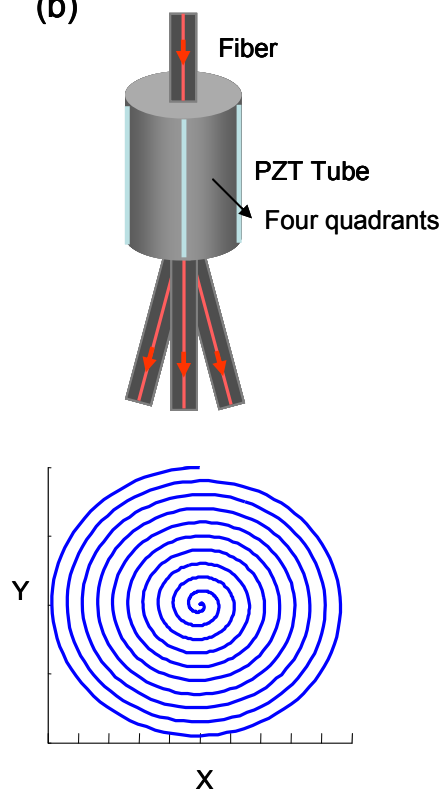

Fig. 3. Schematic of miniature 2D scanning mechanism and pattern: (a) MEMS mirror; (b) PZT based fiber resonant scanner.

Although MEMS scanners can achieve a large lateral beam scan at a high speed, they require complicated fabrication processes and control mechanisms. A simpler method has been developed by scanning the distal tip of an optical fiber cantilever at its mechanical resonant frequency with a piezoelectric actuator (Cobb et al., 2005; Engelbrecht et al., 2008; Flusberg 
et al., 2005b; Helmchen et al., 2001; Liu et al., 2004; Myaing et al., 2006; Seibel \& Smithwick, 2002; Wu et al., 2009a; Wu et al., 2009b). When a piezo actuator is driven at the mechanical resonant frequency of the attached fiber cantilever, small vibration at the cantilever base (on the order of a few microns) can be dramatically amplified at the sweeping fiber tip and a mechanical scanning angle larger than 40 degrees can be conveniently achieved. One type of resonant fiber-optic piezoelectric scanners is based on tubular piezoelectric actuators, as shown in Fig. 3(b) (Engelbrecht et al., 2008; Myaing et al., 2006; Wu et al., 2009a; Wu et al., $2009 \mathrm{~b})$. The cylindrical symmetry makes this type of actuators very suitable for endomicroscopic settings. The outer surface of the PZT tube is divided into four quadrants, forming two pairs of drive electrodes. The fiber-optic cantilever is glued to the actuator tip. One-dimensional resonant line scan is achieved when one pair of electrodes are driven with a sinusoidal waveform at the mechanical resonant frequency of the cantilever, which is given by (Kinsler et al., 1982)

$$
f=\frac{\beta}{4 \pi}\left(\frac{E}{\rho}\right)^{1 / 2}\left(\frac{R}{L^{2}}\right)
$$

Here $f$ is the resonant frequency of the cantilever; $\beta$ is a constant of 3.52 at the zeroth-order vibration mode; E and $\rho$ are the Young's modulus and mass density of the cantilever, respectively; $\mathrm{R}$ and $\mathrm{L}$ are the radius and length of the cantilever, respectively. The equation tells that the cantilever tip scanning frequency is inversely proportional to the square of the cantilever length. For a single-mode fiber cantilever, the rule of thumb is that a $10-\mathrm{mm}$ long cantilever corresponds to a resonant scanning frequency of about $1 \mathrm{kHz}$. The deflection angle of the fiber cantilever $(\alpha)$ is determined by the PZT drive voltage and frequency. The deflection range of the fiber tip is dependent on the length of the fiber, i.e., 2Lsin $\alpha$. The sweeping fiber tip is imaged onto the sample by a micro lens, producing lateral beam scanning. Finally, the lateral beam scanning range $\mathrm{D}$ on the sample is given by

$$
D=2 L M \sin \alpha
$$

where $\mathrm{M}$ is the magnification of the lens from the fiber tip to the sample. When using a 8.5$\mathrm{mm}$ long fiber cantilever and a lens with a magnification of $\sim 1.8$, an approximately $2.5 \mathrm{~mm}$ scanning range on the sample can be achieved with a peak-to-peak drive voltage of $\sim 60 \mathrm{~V}$ near the resonance frequency of $1.4 \mathrm{kHz}$ (Liu et al., 2004).

To generate a circular scan, one pair of the electrodes will be driven with a sine wave while the other pair driven by a cosine wave with the same amplitude and frequency. By triangularly modulating the drive voltage, an open-close spiral scanning pattern can be formed (see Fig. 3(b)). The method has been successfully adopted in TPF endomicroscopes with a frame rate of $2.6-3.3 \mathrm{~Hz}$ composing of $512 \times 512$ pixels with a scanning range of 100 $220 \mu \mathrm{m}$ (Myaing et al., 2006). Using the same method, a TPF endomicroscope prototype has been developed with a video rate of $25 \mathrm{~Hz}$ imaging speed, but with lower sampling pixels (128x128 pixels for a field of view of $200 \mu \mathrm{m})$ (Engelbrecht et al., 2008). Another kind of resonant PZT scanners utilizes a bi-morph piezoelectric plate to vibrate a freely-standing fiber-optic cantilever in Lissajous scanning pattern (Flusberg et al., 2005b; Helmchen et al., 2001). This resonant fiber scanner can also achieve large lateral deflections at the fiber tip (e.g. $\sim 1 \mathrm{~mm}$ ) and a good frame rate of $2 \mathrm{fps}$ with a reasonably compact form (e.g. $8 \mathrm{~mm}$ long x $2 \mathrm{~mm}$ wide $\mathrm{x} 0.5 \mathrm{~mm}$ thick) (Helmchen et al., 2001). 


\subsection{Miniature objective lens}

Another key component in a fiber-optic TPF endomicroscope is the high-quality miniature imaging optics to handle the large separation between the NIR excitation and visible TPF wavelengths. In addition to a compact size, the objective lens should have good optical performance over a broad spectral range to achieve a tight excitation focus (i.e., high lateral and axial resolution as well as excitation efficiency) and reduce chromatic aberration (to prevent the loss in coupling the TPF signals back to the fiber-optic endomicroscope). Gradient-index (GRIN) lenses, owning to their small diameter and cylindrical geometry, have been commonly used as the focusing unit in TPF endomicroscopes (Flusberg et al., 2005b; Fu et al., 2007; Fu et al., 2006; Wu et al., 2009a). Generally, a GRIN objective lens with a diameter of $0.35-3 \mathrm{~mm}$ and a NA of $0.3 \sim 0.6$, can produce lateral and axial resolutions (FWHM) at an order of 1-2 $\mu \mathrm{m}$ and 5-20 $\mu \mathrm{m}$, respectively. However, a GRIN lens suffers severe chromatic aberration, causing a considerable focal shift between the NIR excitation and the visible TPF signals. For a compact, single fiber configuration, a large focal shift will result in dramatic reduction in the TPF collection efficiency (Wu et al., 2009b).

(a)
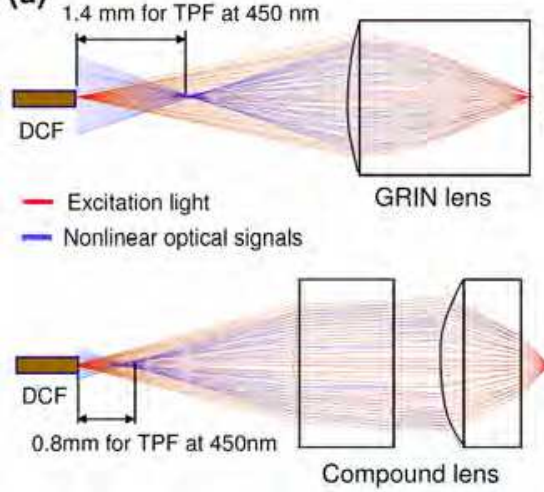

(b)

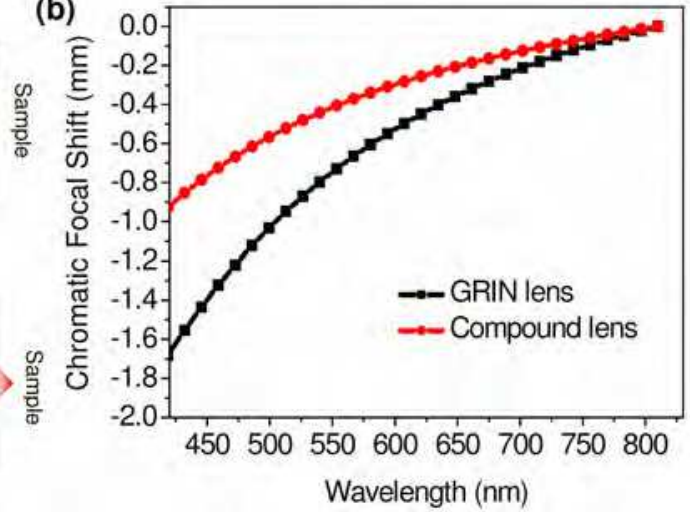

Fig. 4. (a) Representative optical ray plots for excitation light and nonlinear optical signals when using a GRIN lens and an aspherical compound lens; (b) Chromatic longitudinal focal shift from the DCF tip for the TPF signals with $810 \mathrm{~nm}$ excitation when using a GRIN lens and an aspherical compound lens. Images are adapted from Ref. (Wu et al., 2009b) with permission.

A miniature compound lens with less chromatic aberration compared to a GRIN lens, has been proposed and employed in a fiber-optic TPF endomicroscope to increase the TPF collection ( $\mathrm{Wu}$ et al., 2009b). The compound lens was made of a pair of aspherical lenses (2.2-mm effective diameter) with an overall magnification of 0.32 from the fiber tip to the sample. Ray tracing analyses were performed for the compound lens and compared with a GRIN lens (of a $1.8 \mathrm{~mm}$ diameter) for quantitative description of the adverse effect of chromatic aberration in a TPF endomicroscope. Both configurations have the same magnification of 0.32 and the same NA of 0.5 on the sample. Fig. 4(a) shows representative ray plots for excitation light at $810 \mathrm{~nm}$ (in the forward direction, i.e. from the DCF to the sample) and the TPF signals around $450 \mathrm{~nm}$ (in the backward direction). As can be seen, the back focus of the TPF signals from the excitation focal volume within the sample falls in 
front of the DCF tip due to the lens chromatic aberration. Fig. 4(b) quantitatively describes the chromatic longitudinal focal shift from the DCF tip at the TPF wavelengths with an excitation wavelength at $810 \mathrm{~nm}$. It is found that the back focal points of TPF signals (420$600 \mathrm{~nm}$ ) when using a GRIN lens are $\sim 1.7-0.6 \mathrm{~mm}$ in front of the DCF tip, which will dramatically reduce the TPF collection efficiency. In comparison, the aspherical compound lens exhibits less chromatic aberration (e.g. with an $\sim 0.9-0.3 \mathrm{~mm}$ chromatic longitudinal focal shift over 420-600 nm), resulting in an increased TPF collection efficiency.

Table 2 lists the collection efficiency calculated by ray tracing for TPF signals at some typical wavelengths - 450, 500, 550 and $600 \mathrm{~nm}$ when using a GRIN lens and a compound lens, where $100 \%$ representing full collection of the back-reflected TPF signals by a perfect achromatic lens. Here, assuming the collection is based on the commercial double-clad fiber (Fibercore Ltd., SMM900) with an inner clad of 103 um diameter and 0.24 NA. The quantitative analyses indicate that the compound lens increases the collection efficiency of the DCF over the GRIN lens by a factor $\sim 4-6$ for the TPF signals. This simulation result has been confirmed by the experiments on TPF imaging of fluorescent beads (Wu et al., 2009b). It is expected that objective lens with a smaller diameter, higher NA and less chromatic aberration can be fabricated to further improve the excitation and collection efficiency of fiber-optic TPF endomicroscopes.

\begin{tabular}{|c|c|c|c|c|}
\hline $\begin{array}{l}\text { Different Configurations } \\
\text { (810 nm excitation) }\end{array}$ & $\begin{array}{c}\mathrm{TPF} \\
(600 \mathrm{~nm})\end{array}$ & $\begin{array}{c}\mathrm{TPF} \\
(550 \mathrm{~nm})\end{array}$ & $\begin{array}{c}\text { TPF } \\
(500 \mathrm{~nm})\end{array}$ & $\begin{array}{c}\mathrm{TPF} \\
(450 \mathrm{~nm})\end{array}$ \\
\hline GRIN and DCF & $21.5 \%$ & $9.9 \%$ & $5.3 \%$ & $2.1 \%$ \\
\hline Compound Lens and DCF & $78.3 \%$ & $45.7 \%$ & $22.9 \%$ & $12.7 \%$ \\
\hline
\end{tabular}

Table 2. Calculated collection efficiency of TPF signals based on geometrical ray tracing.

To achieve accurate depth-resolved imaging in fiber-optic TPF endomicroscopy, a built-in mechanism for changing the focal plane has to be implemented (preferably at the distal end). A hydraulic system has been demonstrated to move the end of a fiber relative to the objective lens (Rouse et al., 2004). An alternative approach is to utilize a miniature motor to alter the distance between the fiber and the objective lens (Flusberg et al., 2005b). In addition, deformable MEMS lenses and reflecting membranes have also demonstrated a great potential for varying the beam focus at the distal end of the probe by using electrowetting or electrostatic force (Berge \& Peseux, 2000; Kwon et al., 2002). Overall, many challenges remain with distal end beam focus tracking (or depth scanning) in endomicroscopy including system miniaturization, reduction in the drive voltage and improvement on focus tracking accuracy and repeatability over a sufficient range.

\section{A new generation two-photon fluorescence endomicroscopy system}

By addressing the above major challenges for fiber-optic TPF endomicroscope devices, extremely compact all-fiber-optic scanning endomicroscopy systems have been developed for two-photon fluorescence imaging of biological samples (Wu et al., 2009a; Wu et al., 2009b). A conventional DCF was employed for single-mode fs pulse delivery and multimode collection of TPF signals. The DCF also serve as a resonant cantilever driven by a tubular PZT actuator for fast 2D beam scanning. A single PBF with anomalous dispersion at $\sim 810 \mathrm{~nm}$ was used for pulse prechirping in replacement of a bulky grating/lens-based 
pulse stretcher to compensate the normal dispersion of the DCF. The combination of DCF and $\mathrm{PBF}$ in the endomicroscopy system made the system all-fiber-optic and basically a plugand-play unit. For beam focusing, a miniature compound lens was developed to achieve a tight excitation focus, reduce chromatic aberration and improve the excitation and collection efficiency of TPF signals. In addition, a short multimode fiber was introduced at the tip of the DCF to further mitigate the adverse effect of chromatic aberration. Ex vivo TPF imaging of cells and various tissues samples including pig cornea and rat oral cavity was conducted along with this extremely compact all-fiber-optic TPF endomicroscopy system. The imaging results strongly suggest this newly developed endomicroscopy technology has the great potential for in vivo and real-time superficial tissue imaging.

\subsection{Scanning fiber-optic TPF endomicroscope probe}

Fig. 5(a) shows the schematic of the endomicroscope probe. The fiber-optic probe basically consisted of a conventional double-clad fiber, a small tubular piezoelectric actuator and a micro lens for beam focusing. A 300- $\mu \mathrm{m}$ long MMF (105/125 $\mu \mathrm{m}$ core/clad and $0.22 \mathrm{NA})$ was spliced at the distal end of the DCF. The length $(300-\mu \mathrm{m})$ of the MMF collector was chosen such that it would shorten the distance between its end surface and the back focus of the TPF signals as much as possible. Here, the MMF had almost the same core size and NA as the inner clad of the DCF and its short length did not significantly influence the excitation beam path. As confirmed with ray-tracing simulations and experiments on fluorescent bead imaging, the short MMF collector guided more TPF signals back to the DCF.
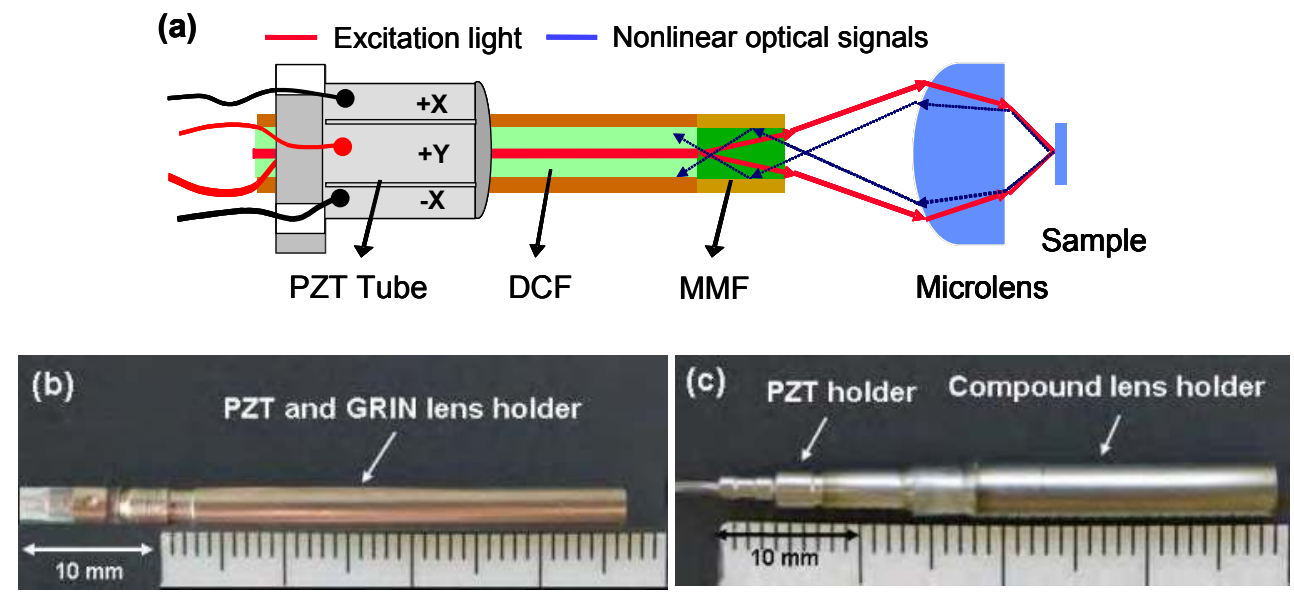

Fig. 5. (a) Schematic of the distal end of the fiber-optic scanning TPF endomicroscope; (b) Photo of a prototype with a GRIN lens; (c) Photo of a prototype with a compound lens. The tubular piezoelectric actuator (PZT), fiber-optic cantilever, and microlens were encased in a hypodermic tube with an overall outer diameter of $2.4 \mathrm{~mm}$ and $3.5 \mathrm{~mm}$ for the GRIN lens and compound lens, respectively. Image (b) is adapted from Ref. (Wu et al., 2009a) with permission; and Image (c) is adapted from Ref. (Wu et al., 2009b) with permission.

For beam focusing, two kinds of miniature optics were implemented, i.e., a GRIN lens and a compound lens, respectively. The GRIN lens with a 0.22 pitch and a 1.8-mm diameter (NSG America, Inc.) had a magnification of 0.5 from the DCF tip to the sample. The compound 
lens was made of a pair of 3-mm miniature aspherical lenses (modified from Archer OpTx L150 and L110 lenses). The purpose of the use of the miniature compound lens is to achieve a tighter focus and increase the TPF excitation efficiency, as well as to minimize the chromatic aberration and increase the TPF collection efficiency.

The endomicroscope had a resonant scanning frequency of $\sim 1,690 \mathrm{~Hz}$ (i.e. 1,690 scanning circles per second would be generated), resulting in a frame rate of 3.3 frames/second with each frame consisting of 512 circular scans. An $\sim 320-\mu \mathrm{m}$ scanning diameter traced by the DCF tip could be achieved with a relatively low peak-to-peak drive voltage of $\sim \pm 30 \mathrm{~V}$. This corresponded to a beam scanning area of $\sim 160 \mu \mathrm{m}$ and $\sim 100 \mu \mathrm{m}$ over the sample when using the GRIN lens with a magnification of 0.5 and the compound lens with a magnification of 0.32 , respectively.

The distal end of the probe, including the piezoelectric actuator, fiber scanner and the lenses, was housed in a thin-wall hypodermic tube with an overall diameter of $2.4 \mathrm{~mm}$ (with the GRIN lens) and $3.5 \mathrm{~mm}$ (with the compound lens), as shown in Fig. 5(b) and Fig. 5(c), respectively. Fig. 6(a) and (b) respectively show a photo of the entire fiber-optic scanning TPF endomicroscope along and a photo of the $2.4 \mathrm{~mm}$ endomicroscope going through the accessory port $(3.8 \mathrm{~mm})$ of a standard endoscope.
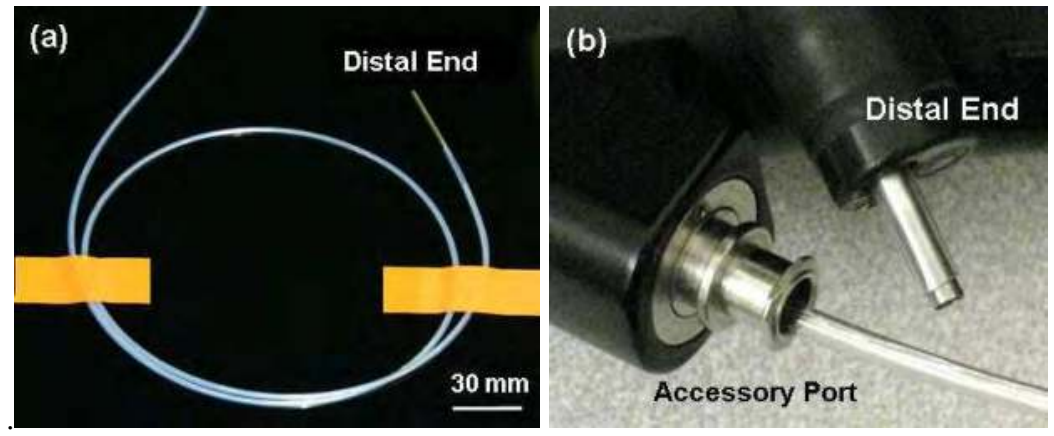

Fig. 6. (a) Photo of the entire fiber-optic scanning TPF endomicroscope probe based on a GRIN lens; (b) Photo of the entire probe with a standard endoscope showing that the fiberoptic endomicroscope can go through the accessory port of a standard endoscope.

\subsection{Entire all-fiber-optic TPF endomicroscopy system}

Fig. 7(a) shows the schematic of the entire endomicroscopy system with a home-built Ti:Sapphire laser. The laser pulses (of a center wavelength $\sim 810 \mathrm{~nm}$, a bandwidth $\sim 36 \mathrm{~nm}$ and a measured temporal pulse width $\sim 60$ fs at the laser output) were coupled into PBF (Crystal Fibers Ltd., HC-800-02) by a fiber-launching lens with an effective NA of $\sim 0.2$ and then directed into the core of the DCF. Here, the PBF was used to compensate the temporal pulse broadening caused by the positive dispersion of femtosecond pulses in the singlemode core of the DCF. The length ratio of the PBF to DCF was kept at $\sim 1.1$ to achieve optimal dispersion compensation for this excitation band $(810 \pm 18 \mathrm{~nm})$ as previously discussed (Wu et al., 2009a). A pulse width of less than $200 \mathrm{fs}$ was achieved even at a power of 50-70 $\mathrm{mW}$ delivered through the DCF core. In addition to its compactness (compared to a grating-lens based pulse stretcher), a single PBF also greatly simplified the system alignment and improved the system stability. Furthermore, the PBF helped to reshape the laser beam 
from the laser output, thus improving the coupling efficiency from the PBF to the DCF core by almost a factor of 2 compared to the use of a pulse stretcher (i.e. from $20-30 \%$ with a pulse stretcher to $\sim 50 \%$ with a PBF). PBF works conveniently for dispersion compensation when the excitation wavelength is fixed to avoid the need for changing the length of the $\mathrm{PBF}$, which is ideal when an extremely compact fs fiber laser is used for excitation. This simple dispersion compensation approach made the entire nonlinear endomicroscopy system compact, reliable and essentially plug-and-play. As shown in Fig. 7(b), the entire endoscope system, including all the fiber-launchers, dispersion unit and the photon detector (except the excitation light source), can be put together within a box of a small footprint (53 $\times 38 \times 18 \mathrm{~cm}$ ), leaving the flexible scanning endomicroscope probe extending outside the box and freely accessible. The compact size and the plug-and-play feature greatly simply the operation of the endomicroscope system and facilitate their applications for both laboratory research and future in vivo studies.
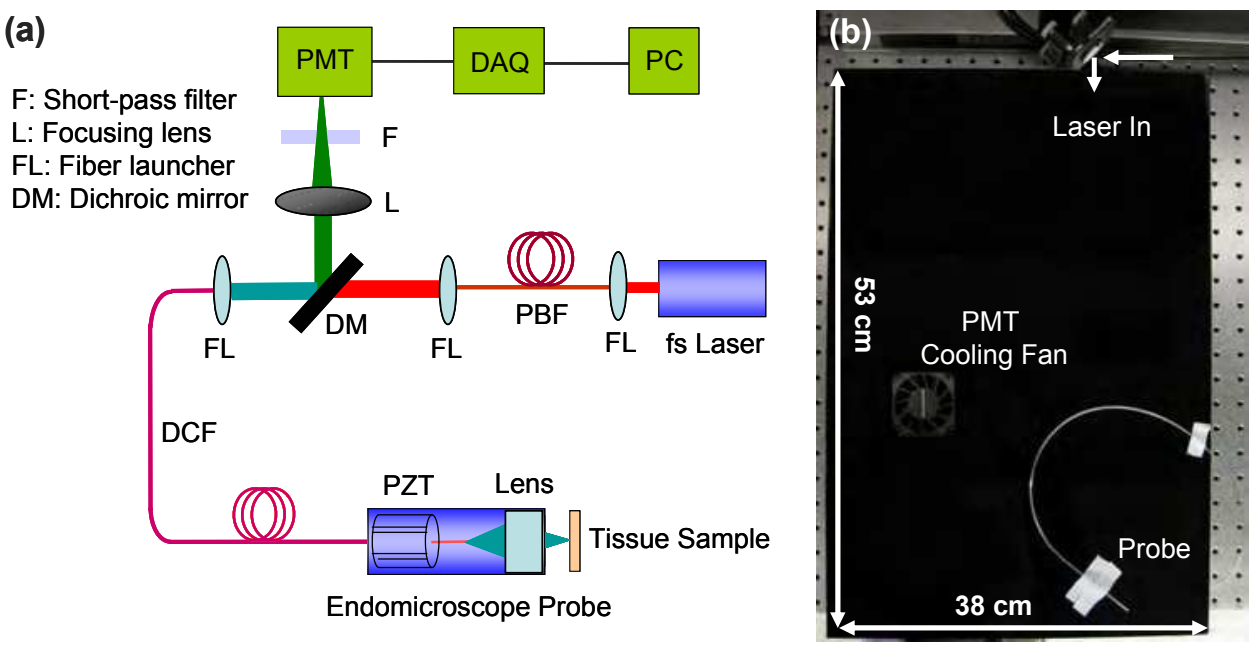

Fig. 7. (a) Schematic of the all-fiber-optic scanning TPF endomicroscope imaging system. The combination of a DCF and PBF made the system all-fiber-optic, in which the DCF was used for both optical nonlinear excitation and TPF (or second harmonic generation) collection, whereas the PBF was employed for dispersion management. PBF: Photonic bandgap fiber; DCF: Double-clad fiber; PMT: Photomultiplier tube; DAQ: Data acquisition. (b) Photo of the all-fiber-optic scanning TPF endomicroscope imaging system inside a box with the flexible endomicroscope probe extended outside the box and placed on the cover. The endomicroscope can be easily plugged into the system and becomes freely accessible.

In imaging experiments, the laser power delivered to the samples was controlled at about 10-15 mW for TPF imaging of stained biological samples. The TPF signals were collected back through the same DCF (core and inner clad), separated from the excitation light at the proximal end of the endomicroscope by a dichroic mirror and then directed to a photomultiplier tube (PMT). The residual excitation light was further blocked by a shortpass filter mounted in front of the PMT. Appropriate band-pass filters were also employed for the detection of the TPF signals. The photo current from the PMT was amplified, converted to voltage and digitized. 
Fluorescent beads were used to test the system performance. Using a phantom made of 0.1$\mu \mathrm{m}$ fluorescent beads, the lateral and axial resolution (FWHM) was measured by scanning through the center of a single bead along the lateral and axial direction, respectively. Gaussian fits to the fluorescence intensities show that the endomicroscopy system with a GRIN lens has $\sim 1.6 \mu \mathrm{m}$ lateral resolution and $\sim 11.4 \mu \mathrm{m}$ axial resolution, whereas the endomicroscopy system with the compound lens has $\sim 1.2 \mu \mathrm{m}$ lateral resolution and $\sim 9.0 \mu \mathrm{m}$ axial resolution, as shown in Figs. 9(a) and (b). The theoretical lateral resolution (FWHM) and axial resolution (FWHM) can be calculated by (Zipfel et al., 2003):

$$
\begin{aligned}
& \text { Lateral resolution }(\mathrm{FWHM})=D \times M \times \sqrt{\ln 2} / 2, \\
& \text { Axial resolution }(\mathrm{FWHM})=D^{2} \times M^{2} \times \sqrt{2} \pi / 4 \lambda,
\end{aligned}
$$

where $\mathrm{D}$ is the mode-filed diameter of the $\mathrm{DCF}, \mathrm{M}$ is the magnification of the endomicroscope from the fiber tip to sample, and $\lambda$ is the excitation wavelength. According to the mode-filed diameter of the DCF $(\mathrm{D}=4.1 \mu \mathrm{m})$, the theoretical lateral resolution should be $0.85 \mu \mathrm{m}$ and $0.54 \mu \mathrm{m}$ for the endomicroscope with the GRIN lens $(\mathrm{M}=0.5)$ and the compound lens $(\mathrm{M}=0.32)$, respectively; the theoretical axial resolution should be $5.75 \mu \mathrm{m}$ and $2.36 \mu \mathrm{m}$ for the endomicroscope with GRIN lens and the compound lens, respectively. This discrepancy is probably mainly attributed to the chromatic aberration and geometrical aberration of the imaging optics, which can be improved with customized achromatic objective lenses.

(a)

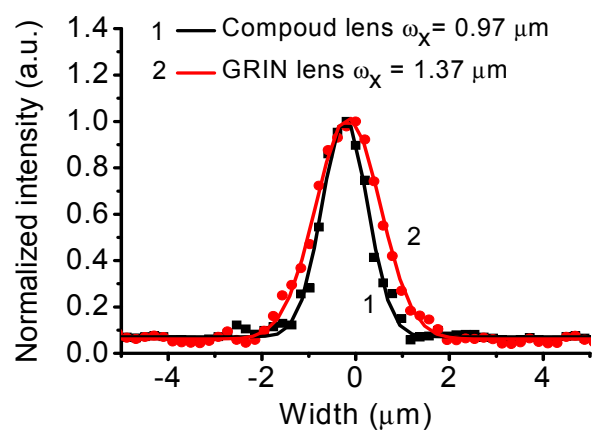

(b)

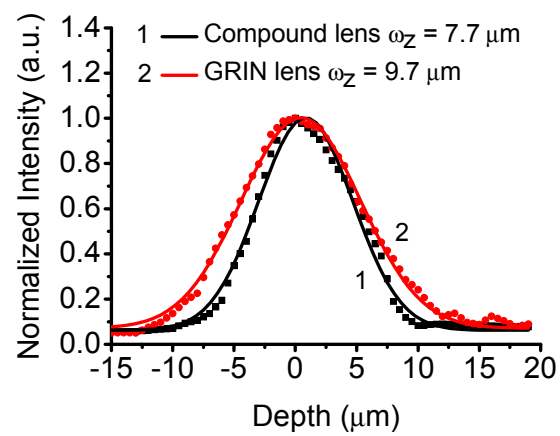

Fig. 8. Fluorescence intensity profiles (dots) across the center of a 0.1- $\mu \mathrm{m}$ fluorescent bead along (a) the lateral and (b) axial dimension. Solid traces are Gaussian-fitted curves.

\subsection{Typical fiber-optic endomicroscopic TPF images}

The imaging capability of the flexible and compact all-fiber-optic scanning endomicroscope was demonstrated on in vitro TPF imaging of stained cancer cells and depth-resolved ex vivo TPF imaging of stained biological samples, as shown in Figs. 9-10, respectively. Fig. 9(a) shows a typical TPF image of breast cancer cells SK-BR-3 that over expressed epidermal growth factor receptors (EGFR). The cells were targeted by fluorescein isothiocyanate (FITC) tagged anti Here2/ $\mathrm{Nu}$ monoclonal antibodies. The single-photon excitation/emission maximum of FITC are 494/521 nm, and the two-photon fluorescence spectrum with 
maximally excitation at $700-850 \mathrm{~nm}$ is similar to the single-photon fluorescence spectrum (Xu et al., 1996). As shown in Fig. 9(a), strong TPF signals of FITC from cell membranes were easily observed. Fig. 9(b) shows a typical TPF image of ovarian cancer cells SK-OV-3. The cells were stained with $\mathrm{AO}$ (acridine orange), the dye for enhancing nuclei contrast (Adams, 1974; Fu et al., 2007). The single- and two-photon fluorescence characteristics of AO when bound to DNA are similar to those of FITC (Neu et al., 2002). As can be seen, cellular nuclei can be clearly identified from the TPF image.
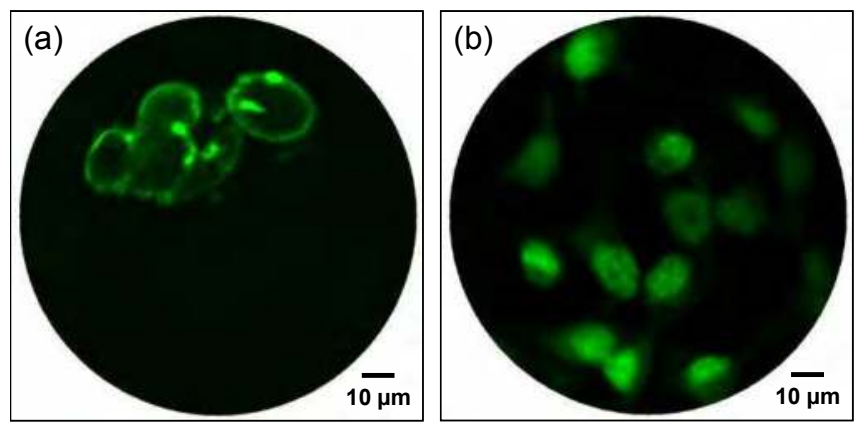

Fig. 9. Typical TPF images obtained with the scanning all-fiber-optic endomicroscope based on the compound lens: (a) Breast cancer cells SK-BR-3 targeted by FITC-tagged monoclonal antibodies (anti Here2/Nu); (b) Ovarian cancer cells SK-OV-3 stained with AO dye; Scale bar: $10 \mu \mathrm{m}$.

Depth-resolved TPF imaging of epithelial tissues was performed using the scanning allfiber-optic endomicroscope based on a GRIN lens, as shown in Fig. 10. All the images were averaged for 10 frames. Figs. 10(a) and (b) show typical images of a formalin fixed pig corneal tissue at $\sim 100 \mu \mathrm{m}$ and $\sim 300 \mu \mathrm{m}$ below the surface, respectively. The tissue sample was stained with $1 \% \mathrm{AO}$ in saline solution after fixation in $10 \%$ formalin. The sparsely distributed keratocytes at different depths can be clearly identified from the images. Figs. $10(\mathrm{c})$ and (d) show representative images of a rat oral tissue sample at depths of 15 and 55 $\mu \mathrm{m}$ below the tissue surface, respectively. The fresh dissected tissue sample was directly stained with 1\% AO and the epithelial cell nuclei can be identified by the TPF images. As seen from the depth-resolved images, the nucleus density increases from the superficial layer to the basal layer. Figs. 10(e) and (f) show typical images of a chick cochlear section expressing GPF (green fluorescence protein). The single-photon excitation/emission maximum of GFP are $491 / 510 \mathrm{~nm}$, whereas its two-photon excitation/emission maxima are about $900 / 510 \mathrm{~nm}$ (Xu et al., 1996). Although the excitation efficiency is relatively weak at $810 \mathrm{~nm}$ excitation, the structures (hair cells) revealed by the TPF signals are still observable. Different distribution of hair cells can be observed at different depths (see Fig. 10(e) from the surface and Fig. 10(f) from $40 \mu \mathrm{m}$ depth below the surface). Overall, the depth-resolved TPF images clearly reveal the tissue layered structures that are consistent with text book histology (Ross et al., 1989).

Overall, the extremely compact, all-fiber-optic and plug-and-play scanning endomicroscopy system has shown excellent ability in two-photon fluorescence imaging of biological samples. The system employed a conventional double-clad fiber for femtosecond pulse delivery, TPF signals collection and fast beam scanning. By replacing a bulky grating/lensbased pulse stretcher with a single photonic bandgap fiber for pulse prechirping, the system 

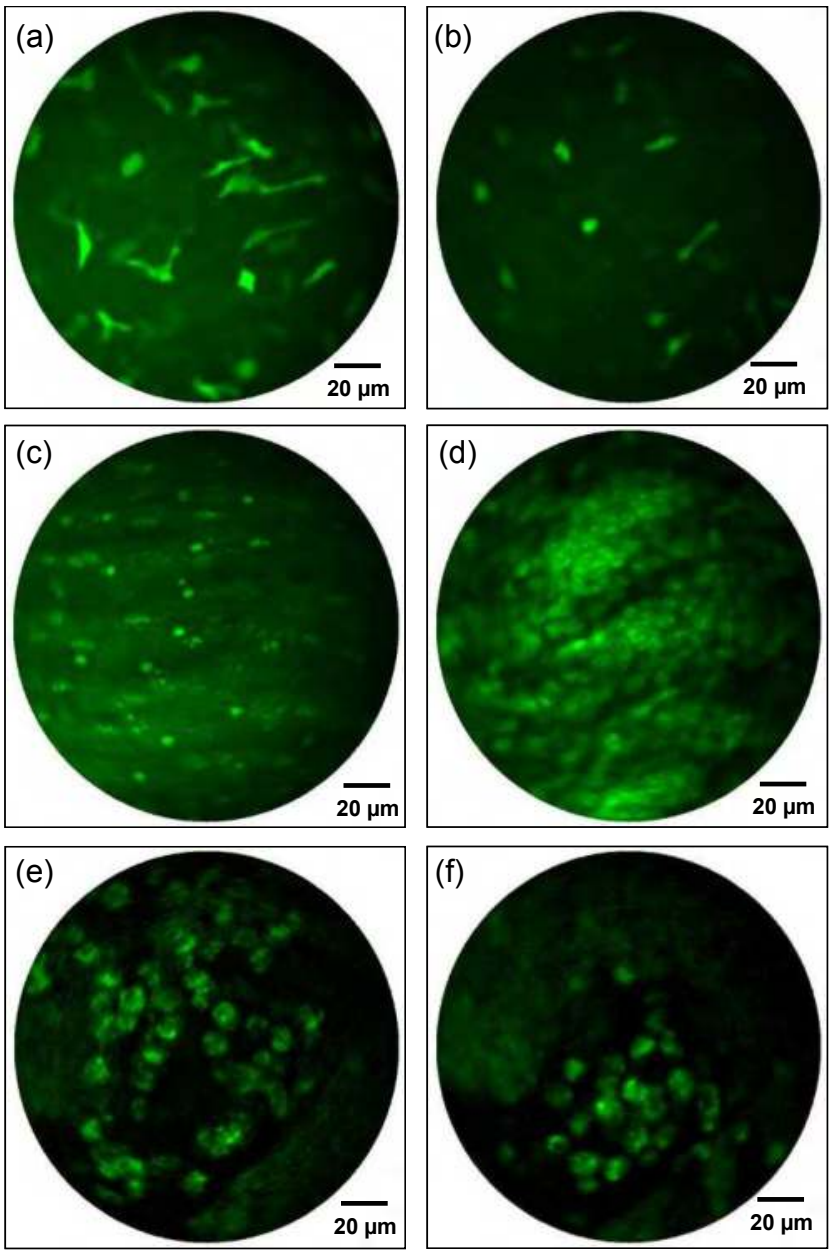

Fig. 10. Typical depth-resolved TPF images obtained with the scanning all-fiber-optic endomicroscope bases on a GRIN lens: ( $a$ \& b) Pig cornea sample stained with AO dye (a) at the depth of $100 \mu \mathrm{m}$ and (b) at the depth of $300 \mu \mathrm{m}$; (c \& d) Rat oral tissue stained with AO dye (c) at the depth of $15 \mu \mathrm{m}$ and (d) at the depth of $55 \mu \mathrm{m}$; (e \& f) Chick cochlear expressing GFP (e) from the tissue surface and (b) at the depth of $40 \mu \mathrm{m}$. Scale bar: $20 \mu \mathrm{m}$.

size is significantly reduced and the power delivery efficiency through the endomicroscope is improved. Real-time ex vivo 2D and depth-resolved TPF imaging of biological samples were performed with the scanning endomicroscope at cellular and subcellular resolution. The preliminary results strongly suggest the promising potential of this all-fiber-optic endomicroscopy technology as a basic laboratory research tool and a clinical tool for realtime assessment of epithelium and stroma under various clinically relevant conditions. The performance of the endomicroscope system can be further improved to increase the signalto-noise ratio and resolve more detailed intracellular structures by introducing better compound lenses with higher NAs. 


\section{Summary}

In this book chapter, the general technological and engineering challenges in developing a scanning two-photon fluorescence fiber-optic endomicroscopy system has been discussed, including single-mode femtosecond laser delivery, large-area TPF signal collection, dispersion management, fast two-dimensional beam scanning and high-quality miniature imaging optics. Detailed design issues and imaging performance has been illustrated using our recently developed scanning all-fiber-optic TPF endomicroscope as an example. The two-photon fluorescence endomicroscope has shown great flexibility and reliability for high-resolution imaging of internal luminal organs.

In summary, endomicroscopy technologies for two-photon fluorescence imaging have been under rapid development, aiming for non-invasive, high-resolution and high-speed imaging of tissue microstructures and assessment of tissue pathology in vivo. The development of optical fibers (such as double-clad fibers and photonic bandgap fibers), miniature beam scanning mechanisms (such as MEMS scanners and fiber-optic resonant scanners) and miniature imaging optics (such as compound lens with chromatic aberration and high NA) greatly facilitates probe miniaturization and functional integration for high-resolution and high-speed TPF imaging of biological samples. With further technology innovation, miniature fiber-optic TPF endomicroscope can potentially generate high-quality images approaching to those achieved by standard bench-top two-photon fluorescence microscopy in the near future.

\section{Acknowledgement}

The authors greatly thank the invaluable contributions of Dr. Michael J. Cobb, Dr. Yuxin Leng, Daniel J. MacDonald, Jeff Magula, Dr. Mon T. Myaing and Jiefeng $\mathrm{Xi}$ in the development of the fiber-optic two-photon fluorescence endomicroscopy technologies. This work has been supported in part by the National Institutes of Health and National Science Foundation (Career Award XDL).

\section{References}

Adams, L.R. (1974). Acridine-Orange Staining of Epithelial-Cells in Strong Salt Solution. Journal of Histochemistry \& Cytochemistry, Vol. 22, No. 7, pp. 492-494.

Agrawal, G.P. (2007). Nonlinear Fiber Optics. Academic Press: San Diego.

Bao, H.C.; Allen, J.; Pattie, R.; Vance, R. \& Gu, M. (2008). Fast handheld two-photon fluorescence microendoscope with a $475 \mathrm{mu} \mathrm{m}$ X $475 \mathrm{mu}$ m field of view for in vivo imaging. Optics Letters, Vol. 33, No. 12, pp. 1333-1335.

Bao, H.C. \& Gu, M. (2009). Reduction of self-phase modulation in double-clad photonic crystal fiber for nonlinear optical endoscopy. Optics Letters, Vol. 34, No. 2, pp. 148150.

Berge, B. \& Peseux, J. (2000). Variable focal lens controlled by an external voltage: An application of electrowetting. European Physical Journal E, Vol. 3, No. 2, pp. 159-163.

Bjarklev, A.; Broeng, J. \& Bjarklev, A.S. (2003). Photonic Crystal Fibers. Kluwer Academic Publishers: Norwell, MA.

Cobb, M.J.; Liu, X.M. \& Li, X.D. (2005). Continuous focus tracking for real-time optical coherence tomography. Optics Letters, Vol. 30, No. 13, pp. 1680-1682. 
Denk, W.; Strickler, J.H. \& Webb, W.W. (1990). 2-Photon Laser Scanning Fluorescence Microscopy. Science, Vol. 248, No. 4951, pp. 73-76.

Engelbrecht, C.J.; Johnston, R.S.; Seibel, E.J. \& Helmchen, F. (2008). Ultra-compact fiber-optic two-photon microscope for functional fluorescence imaging in vivo. Optics Express, Vol. 16, No. 8, pp. 5556-5564.

Flusberg, B.A.; Cocker, E.D.; Piyawattanametha, W.; Jung, J.C.; Cheung, E.L.M. \& Schnitzer, M.J. (2005a). Fiber-optic fluorescence imaging. Nature Methods, Vol. 2, No. 12, pp. 941-950.

Flusberg, B.A.; Lung, J.C.; Cocker, E.D.; Anderson, E.P. \& Schnitzer, M.J. (2005b). In vivo brain imaging using a portable 3.9 gram two-photon fluorescence microendoscope. Optics Letters, Vol. 30, No. 17, pp. 2272-2274.

Fu, L.; Gan, X.S. \& Gu, M. (2005). Nonlinear optical microscopy based on double-clad photonic crystal fibers. Optics Express, Vol. 13, No. 14, pp. 5528-5534.

Fu, L.; Jain, A.; Cranfield, C.; Xie, H.K. \& Gu, M. (2007). Three-dimensional nonlinear optical endoscopy. Journal of Biomedical Optics, Vol. 12, No. 4, pp. 040501.

Fu, L.; Jain, A.; Xie, H.K.; Cranfield, C. \& Gu, M. (2006). Nonlinear optical endoscopy based on a double-clad photonic crystal fiber and a MEMS mirror. Optics Express, Vol. 14, No. 3, pp. 1027-1032.

Gobel, W.; Kerr, J.N.D.; Nimmerjahn, A. \& Helmchen, F. (2004a). Miniaturized two-photon microscope based on a flexible coherent fiber bundle and a gradient-index lens objective. Optics Letters, Vol. 29, No. 21, pp. 2521-2523.

Gobel, W.; Nimmerjahn, A. \& Helmchen, F. (2004b). Distortion-free delivery of nanojoule femtosecond pulses from a $\mathrm{Ti}$ : sapphire laser through a hollow-core photonic crystal fiber. Optics Letters, Vol. 29, No. 11, pp. 1285-1287.

Hagelin, P.M. \& Solgaard, O. (1999). Optical raster-scanning displays based on surfacemicromachined polysilicon mirrors. Ieee Journal of Selected Topics in Quantum Electronics, Vol. 5, No. 1, pp. 67-74.

Hah, D.; Huang, S.T.Y.; Tsai, J.C.; Toshiyoshi, H. \& Wu, M.C. (2004). Low-voltage, large-scan angle MEMS analog micromirror arrays with hidden vertical comb-drive actuators. Journal of Microelectromechanical Systems, Vol. 13, No. 2, pp. 279-289.

Helmchen, F. \& Denk, W. (2005). Deep tissue two-photon microscopy. Nature Methods, Vol. 2, No. 12, pp. 932-940.

Helmchen, F.; Fee, M.S.; Tank, D.W. \& Denk, W. (2001). A miniature head-mounted twophoton microscope: High-resolution brain imaging in freely moving animals. Neuron, Vol. 31, No. 6, pp. 903-912.

Hoy, C.L.; Durr, N.J.; Chen, P.Y.; Piyawattanametha, W.; Ra, H.; Solgaard, O. \& Ben-Yakar, A. (2008). Miniaturized probe for femtosecond laser microsurgery and two-photon imaging. Optics Express, Vol. 16, No. 13, pp. 9996-10005.

Jung, J.C. \& Schnitzer, M.J. (2003). Multiphoton endoscopy. Optics Letters, Vol. 28, No. 11, pp. 902-904.

Jung, W.Y.; Tang, S.; McCormic, D.T.; Xie, T.Q.; Ahn, Y.C.; Su, J.P.; Tomov, I.V.; Krasieva, T.B.; Tromberg, B.J. \& Chen, Z.P. (2008). Miniaturized probe based on a microelectromechanical system mirror for multiphoton microscopy. Optics Letters, Vol. 33, No. 12, pp. 1324-1326.

Kinsler, L.E.; Frey, A.R.; Coppens, A.B. \& Sanders, J.V. (1982). Fundamentals of Acoustics. Wiley: New York. 
Knight, J.C. (2003). Photonic crystal fibres. Nature, Vol. 424, No. 6950, pp. 847-851.

Konig, K. (2000). Multiphoton microscopy in life sciences. Journal of Microscopy-Oxford, Vol. 200, pp. 83-104.

Konig, K.; Ehlers, A.; Riemann, I.; Schenkl, S.; Buckle, R. \& Kaatz, M. (2007). Clinical twophoton microendoscopy. Microscopy Research and Technique, Vol. 70, No. 5, pp. 398402.

Kwon, S.; Milanovic, V. \& Lee, L.P. (2002). Large-displacement vertical microlens scanner with low driving voltage. Ieee Photonics Technology Letters, Vol. 14, No. 11, pp. 15721574.

Lang, W.; Pavlicek, H.; Marx, T.; Scheithauer, H. \& Schmidt, B. (1999). Electrostatically actuated micromirror devices in silicon technology. Sensors and Actuators a-Physical, Vol. 74, No. 1-3, pp. 216-218.

Le Harzic, R.; Weinigel, M.; Riemann, I.; Konig, K. \& Messerschmidt, B. (2008). Nonlinear optical endoscope based on a compact two axes piezo scanner and a miniature objective lens. Optics Express, Vol. 16, No. 25, pp. 20588-20596.

Levene, M.J.; Dombeck, D.A.; Kasischke, K.A.; Molloy, R.P. \& Webb, W.W. (2004). In vivo multiphoton microscopy of deep brain tissue. Journal of Neurophysiology, Vol. 91, No. 4, pp. 1908-1912.

Lin, H.Y. \& Fang, W.L. (2003). A rib-reinforced micro torsional mirror driven by electrostatic torque generators. Sensors and Actuators a-Physical, Vol. 105, No. 1, pp. 1-9.

Liu, X.M.; Cobb, M.J.; Chen, Y.C.; Kimmey, M.B. \& Li, X.D. (2004). Rapid-scanning forwardimaging miniature endoscope for real-time optical coherence tomography. Optics Letters, Vol. 29, No. 15, pp. 1763-1765.

Myaing, M.T.; MacDonald, D.J. \& Li, X.D. (2006). Fiber-optic scanning two-photon fluorescence endoscope. Optics Letters, Vol. 31, No. 8, pp. 1076-1078.

Neu, T.R.; Kuhlicke, U. \& Lawrence, J.R. (2002). Assessment of fluorochromes for twophoton laser scanning microscopy of biofilms. Applied and Environmental Microbiology, Vol. 68, No. 2, pp. 901-909.

Piyawattanametha, W.; Barretto, R.P.J.; Ko, T.H.; Flusberg, B.A.; Cocker, E.D.; Ra, H.J.; Lee, D.S.; Solgaard, O. \& Schnitzer, M.J. (2006). Fast-scanning two-photon fluorescence imaging based on a microelectromechanical systems two-dimensional scanning mirror. Optics Letters, Vol. 31, No. 13, pp. 2018-2020.

Reeves, W.H.; Skryabin, D.V.; Biancalana, F.; Knight, J.C.; Russell, P.S.; Omenetto, F.G.; Efimov, A. \& Taylor, A.J. (2003). Transformation and control of ultra-short pulses in dispersion-engineered photonic crystal fibres. Nature, Vol. 424, No. 6948, pp. 511515.

Ross, M.H.; Reith, E.J. \& Romrell, L.J. (1989). Histology: A text and atlas. Williams \& Wilkins.

Rouse, A.R.; Kano, A.; Udovich, J.A.; Kroto, S.M. \& Gmitro, A.F. (2004). Design and demonstration of a miniature catheter for a confocal microendoscope. Applied Optics, Vol. 43, No. 31, pp. 5763-5771.

Schenk, H.; Durr, P.; Haase, T.; Kunze, D.; Sobe, U.; Lakner, H. \& Kuck, H. (2000). Large deflection micromechanical scanning mirrors for linear scans and pattern generation. Ieee Journal of Selected Topics in Quantum Electronics, Vol. 6, No. 5, pp. 715-722.

Seibel, E.J. \& Smithwick, Q.Y.J. (2002). Unique features of optical scanning, single fiber endoscopy. Lasers in Surgery and Medicine, Vol. 30, No. 3, pp. 177-183. 
Treacy, E.B. (1969). Optical Pulse Compression with Diffraction Gratings. Ieee Journal of Quantum Electronics, Vol. Qe 5, No. 9, pp. 454-\&.

Wu, Y.C.; Leng, Y.X.; Xi, J.F. \& Li, X.D. (2009a). Scanning all-fiber-optic endomicroscopy system for 3D nonlinear optical imaging of biological tissues. Optics Express, Vol. 17, No. 10, pp. 7907-7915.

Wu, Y.C.; Xi, J.F.; Cobb, M.J. \& Li, X.D. (2009b). Scanning fiber-optic nonlinear endomicroscopy with miniature aspherical compound lens and multimode fiber collector. Optics Letters, Vol. 34, No. 7, pp. 953-955.

Xu, C.; Zipfel, W.; Shear, J.B.; Williams, R.M. \& Webb, W.W. (1996). Multiphoton fluorescence excitation: New spectral windows for biological nonlinear microscopy. Proceedings of the National Academy of Sciences of the United States of America, Vol. 93, No. 20, pp. 10763-10768.

Yao, Z.J. \& MacDonald, N.C. (1997). Single crystal silicon supported thin film micromirrors for optical applications. Optical Engineering, Vol. 36, No. 5, pp. 1408-1413.

Zipfel, W.R.; Williams, R.M. \& Webb, W.W. (2003). Nonlinear magic: multiphoton microscopy in the biosciences. Nature Biotechnology, Vol. 21, No. 11, pp. 1368-1376. 


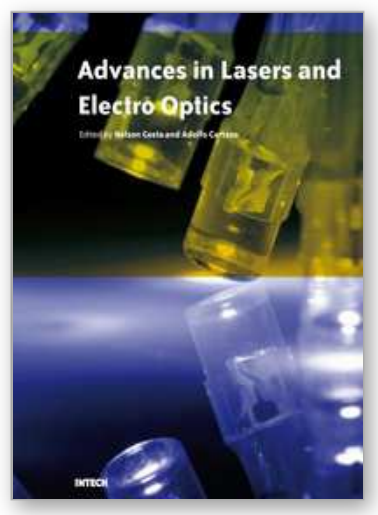

\author{
Advances in Lasers and Electro Optics \\ Edited by Nelson Costa and Adolfo Cartaxo
}

ISBN 978-953-307-088-9

Hard cover, 838 pages

Publisher InTech

Published online 01, April, 2010

Published in print edition April, 2010

Lasers and electro-optics is a field of research leading to constant breakthroughs. Indeed, tremendous advances have occurred in optical components and systems since the invention of laser in the late $50 \mathrm{~s}$, with applications in almost every imaginable field of science including control, astronomy, medicine, communications, measurements, etc. If we focus on lasers, for example, we find applications in quite different areas. We find lasers, for instance, in industry, emitting power level of several tens of kilowatts for welding and cutting; in medical applications, emitting power levels from few milliwatt to tens of Watt for various types of surgeries; and in optical fibre telecommunication systems, emitting power levels of the order of one milliwatt. This book is divided in four sections. The book presents several physical effects and properties of materials used in lasers and electro-optics in the first chapter and, in the three remaining chapters, applications of lasers and electro-optics in three different areas are presented.

\title{
How to reference
}

In order to correctly reference this scholarly work, feel free to copy and paste the following:

Yicong Wu and Xingde Li (2010). Two-photon Fluorescence Endomicroscopy, Advances in Lasers and Electro Optics, Nelson Costa and Adolfo Cartaxo (Ed.), ISBN: 978-953-307-088-9, InTech, Available from: http://www.intechopen.com/books/advances-in-lasers-and-electro-optics/two-photon-fluorescenceendomicroscopy

\section{INTECH}

open science | open minds

\section{InTech Europe}

University Campus STeP Ri

Slavka Krautzeka 83/A

51000 Rijeka, Croatia

Phone: +385 (51) 770447

Fax: +385 (51) 686166

www.intechopen.com

\section{InTech China}

Unit 405, Office Block, Hotel Equatorial Shanghai

No.65, Yan An Road (West), Shanghai, 200040, China 中国上海市延安西路65号上海国际贵都大饭店办公楼 405 单元

Phone: +86-21-62489820

Fax: $+86-21-62489821$ 
(C) 2010 The Author(s). Licensee IntechOpen. This chapter is distributed under the terms of the Creative Commons Attribution-NonCommercialShareAlike-3.0 License, which permits use, distribution and reproduction for non-commercial purposes, provided the original is properly cited and derivative works building on this content are distributed under the same license. 